\title{
MISURE DI AUSTERITÀ E DISCRIMINAZIONE SULLA BASE DELLA DISABILITÀ: UNA RECENTE DECISIONE DELLA CORTE DI STRASBURGO
}

\author{
di Silvia Favalli
}

\section{Austerità, discriminazione e vulnerabilità}

Le misure di austerità messe in campo da molti paesi europei a seguito della ben nota crisi economica del 2008 sono state, negli ultimi anni, non solo oggetto di critica politica, ma anche sottoposte allo scrutinio della Corte europea dei diritti dell'uomo (Corte EDU) per violazione dei diritti fondamentali dell'individuo. In particolare, in più di un'occasione, i giudici di Strasburgo sono stati chiamati a giudicare la compatibilità di tali restrizioni con i diritti sanciti all'interno della Convenzione europea dei diritti dell'uomo (CEDU) ${ }^{1}$. Se, da un lato, la Corte EDU ha sempre riconosciuto un ampio margine di apprezzamento in capo agli Stati contraenti nell'attuazione delle politiche nazionali di sicurezza sociale ${ }^{2}$, dall' altro, ha però utilizzato il principio di non discri-

Assegnista di ricerca in Diritto internazionale presso il Dipartimento di Scienze Politiche e Sociali dell'Università di Pavia. Questo scritto si inserisce nell'ambito delle attività di ricerca sviluppate nel quadro del progetto "RISID Realizing the right to Social Inclusion for persons with Disabilities through new tools of smart communication and sharing knowledge: from international to local effectiveness", finanziato da Fondazione Cariplo.

1 Vedi O. P. VAsYlchenko, O.S. Lotiuk, Topical issues of the impact of austerity measures on human rights: the case of Europe, in "Financial and credit activity: problems of theory and practice", n. 3, 2018, pp. 477-483; C. O'CINNEIDE, Austerity and the faded dream of a social Europe, in A. Nolan, Economic and Social Rights after the Global Financial Crisis, OUP, 2014, pp. 169-201; J. GERARDS, The ECtHR's Response to Fundamental Rights Issues Related to Financial and Economic Difficulties: the Problem of Compartmentalisation, in "Netherlands Quarterly of Human Rights", n. 3, 2015, pp. 274-292.

2 L. Mola, The margin of appreciation accorded to States in times of economic crisis: an analysis of the decisions by the European Committee of Social Rights and by the Euro- 
minazione come limite all'imposizione di misure di austerità a particolare discapito di determinate categorie di individui, per i quali, proprio a causa della loro peculiare condizione di vulnerabilità, esse potrebbero rivelarsi eccessivamente gravose 3 .

Sulla base di queste considerazioni, il presente contributo si propone di analizzare criticamente una recente pronuncia della Corte EDU, J.D. e A. c. Regno Unito 4 , la quale ha, per la prima volta, messo in discussione le politiche di austerity adottate in materia di sussidi abitativi dal governo inglese nel 2012 a detrimento di una specifica categoria di soggetti vulnerabili, i.e. le vittime di violenza domestica. È stato sottolineato, in particolare, come il ragionamento della Corte si sia orientato verso criteri improntati al principio di uguaglianza sostanziale piuttosto che formale ${ }^{5}$, aprendo così uno spiraglio verso un nuovo approccio, meno restrittivo, in relazione all'adozione di misure economiche e sociali6. Tale decisione risulta tanto più significativa perché riguarda un paese, il Regno Unito, che, come è stato posto in evidenza dagli osservatori più critici, ha conosciuto, a partire dalla crisi economica del 2008, un percorso di austerità e di "graduale smantellamento delle garanzie sociali" 7 . Un percorso che, peraltro, non sarà destinato probabilmente a regredire nel prossimo futuro,

pean Court of Human Rights on national austerity measures, in "Revistia juridica de los Derechos Sociales", 2015, p. 186 sgg.; F. SAITTO, La "solitudine” delle corti costituzionali? Sindacato sulle misure di austerity e protezione dei diritti sociali tra giudici nazionali e Corte EDU, in "Diritto pubblico", n. 1, 2016, p. 434 sgg.

3 STEERING COMMITTEE FOR HUMAN RIGHTS (CDDH), The impact of the economic crisis and austerity measures on human rights in Europe: Feasibility study, Consiglio d'Europa, Strasburgo, 2016, p. 38, in https://rm.coe.int/the-impact-of-the-economic-crisis-and-austerity-measures-on-human-righ/16806f2030 (consultato il 24 aprile 2020).

4 Corte EDU, J.D. e A. c. Regno Unito, ricorsi n. 32949/17 e 34614/17, 24 ottobre 2019.

5 K. Frostell, Gender-based violence triggers differential treatment in housing benefit case, in "Strasboug Observer", 2 dicembre 2019, in https://strasbourgobservers.com/2019/12/ 02/gender-based-violence-triggers-differential-treatment-in-housing-benefit-case/(consultato il 24 aprile 2020).

6 A. EwING, "Bedroom tax" unlawful - Strasbourg Court, in "UK Human Rights Blog”, 12 novembre 2019, in https://ukhumanrightsblog.com/2019/11/12/bedroom-taxunlawful-strasbourg-court/.

7 B.A. MAsso, Diritti sociali ed economici nel Regno Unito all'epoca della Brexit e di austerity: la critica del Presidente della Corte Suprema, in "Diritto pubblico comparato ed europeo", n. 1, 2020, pp. 273-280. 
vista l'incertezza legata alla Brexit e la crisi economica innestata dalla pandemia covid-19.

Si ricorda inoltre che, nell'ordinamento inglese, le Corti superiori hanno la possibilità, grazie allo Human Rights Act, di pronunciare una declaration of incompatibility di tutti gli atti legislativi primari che vengano giudicati in contrasto con la CEDU, qualora sia esclusa la possibilità di darne un'interpretazione conforme. In assenza di un testo costituzionale scritto e di un sistema giudiziale formalizzato di controllo di costituzionalità, questa operazione è stata indicata come una sorta di sindacato costituzionale, seppur non vincolante per il legislatore $^{8}$, il quale trova nella pronuncia della Corte superiore nazionale le linee guida per eventualmente emendare l'atto legislativo nel rispetto della CEDU. In questo modo, la giurisprudenza delle Corti superiori britanniche si fa "portatrice di istanze di presidio e salvaguardia dei diritti sociali ed economici", usufruendo della CEDU come leva per rafforzare le garanzie sociali ed economiche all'interno del proprio ordinamento nazionale ${ }^{9}$. Ne consegue che la portata innovativa della sentenza in esame è in grado di avere ripercussioni significative sulla futura giurisprudenza nazionale, la quale si adeguerà a quanto in essa statuito proprio in virtù di tale "tecnica costituzionale".

Tuttavia, l'analisi qui proposta adotterà una posizione critica, concentrandosi su quel che si ritiene invece il "tassello mancante" della decisione in esame, vale a dire l'analisi del caso anche alla luce del principio di non discriminazione fondata sulla disabilità. In particolare, considerato che, come si vedrà nel prosieguo, la Convenzione delle Nazioni Unite sui diritti delle persone con disabilità del 2006

8 Secondo Duxbury, la declaration of incompatibility disciplinata nello Human Rights Act del 1998 incarna un esempio di judical disapproval, che l'Autore ritiene "a form of non-binding review of the constitutionality of legislation". Vedi N. DUXBURY, Judicial disapproval as a constitutional technique, in "International Journal of Constitutional Law", n. 3, 2017, pp. 649-670. Kavanagh critica la qualificazione della declaration of incompatibility come una forma di judicial disapproval, ma ne ribadisce "the strong censorial effect" nella cultura giuridica britannica. Vedi A. KaVAnagh, Article Review: Aileen Kavanagh on Neil Duxbury's Judicial Disapproval as a Constitutional Technique, in "Blog of the International Journal of Constitutional Law", 29 novembre 2017, in http://www.iconnectblog. com/2017/11/aileen-kavanagh-on-neil-duxburys-judicial-disapproval-as-a-constitutional-technique/ (consultato il 24 aprile 2020).

9 B.A. MAsso, Diritti sociali ed economici nel Regno Unito all'epoca della Brexite di austerity: la critica del Presidente della Corte Suprema, cit., p. 279. 
(CDPD $)^{10}$ ormai costituisce lo standard normativo di riferimento in materia di tutela dei diritti delle persone con disabilità anche nell'ambito del Consiglio d'Europa, le disposizioni rilevanti della CEDU devono essere lette alla luce del modello di "uguaglianza sostanziale" sancito in tale trattato. Si ritiene, infatti, che, proprio a causa di tale lacuna nel ragionamento della Corte EDU, i giudici di Strasburgo abbiano perso l'opportunità di offrire la stessa tutela rafforzata anche nei confronti di altri individui in situazione di particolare vulnerabilità, quali le persone con disabilità e i loro caregiver.

\section{Il caso J.D. e A. c. Regno Unito}

Il caso in esame trae origine dai ricorsi, successivamente riuniti, di due cittadine britanniche che lamentano di essere state discriminate, rispettivamente, sulla base della disabilità (la signora J.D.) e del genere (la signora A.), dalla normativa nazionale che aveva ridotto il sussidio abitativo di cui antecedentemente godevano per il paga-

${ }^{10}$ La bibliografia che riguarda la CDPD è amplissima. Vedi, fra i molti, F. SEATZU, $L a$ convenzione delle Nazioni Unite sui diritti delle persone disabili: i principi fondamentali, in "Diritti umani e diritto internazionale", n. 3, 2008, pp. 535-559; ID., La convenzione delle Nazioni Unite sui diritti delle persone disabili: diritti garantiti, cooperazione, procedure di controllo, in "Diritti umani e diritto internazionale", 2009, pp. 259-280; F. MÉGRÉT, The Disabilities Convention: towards a holistic concept of right, in "The International Journal of Human Rights", n. 2, 2008, pp. 261-278; ID., The Disabilities Convention: human rights of persons with disabilities or disability rights?, in "Human Rights Quarterly", n. 2, 2008, pp. 494-516; R. KAYNESS, P. French, Out of Darkness into Light? Introducing the Convention on the Rights of Persons with Disabilities under EU Law, in "Human Rights Law Review", n. 1, 2008, pp. 1-34; N. FogGetTI, Diritti umani e tutela delle persone con disabilità: la convenzione delle Nazioni Unite del 13 dicembre 2006, in "Rivista della cooperazione giuridica internazionale", 2009, pp. 98-117; S. MARCHISIO, V. Della Fina, R. CERA, La convenzione delle Nazioni Unite sui diritti delle persone con disabilità: commentario, Roma, Aracne 2010; M. SCHULZE, Understanding the UN convention on the rights of persons with disabilities: a handbook on the human rights of persons with disabilities, New York, 2010, in http://www.hiproweb.org/uploads/tx_hidrtdocs/HICRPDManual2010.pdf (consultato il 4 agosto 2020); V. Della Fina, R. Cera, G. PAlmisano (eds.), The United Nations Convention on the Rights of Persons with Disabilities: A Commentary, Cham, Springer, 2017; C. O'Mahony, G. Quinn, Disability Law and Policy: An Analysis of the UN Convention, Dublino, Clarus Press, 2017; I. Bantekas, M.A. SteIn, D. AnastaSIOU (eds.), The Convention on the Rights of Persons with Disabilities: A Commentary, Oxford, oup, 2018. 
mento del canone di locazione della casa di abitazione, il c.d. House Benefit.

Più precisamente, nel 2012, il governo inglese aveva adottato un emendamento alla normativa di riferimento (c.d. Regulation B13) che riduceva la somma di tale sussidio in base alla presenza di camere in più rispetto al numero degli occupanti effettivi dell'immobile, al fine di incentivare il rilascio delle case con stanze non occupate e razionalizzare l'erogazione di tale misura di assistenza sociale. Come risultato, le due ricorrenti, a cui veniva ridotto tale sussidio del 14\% perché entrambe disponevano di una camera aggiuntiva, si trovavano nell'impossibilità di sostenere il costo del canone di locazione. Le donne facevano anche domanda per ottenere le somme decurtate tramite un altro strumento di sicurezza sociale messo a disposizione dalle autorità nazionali, il c.d. Discretionary Housing Payment (o DHP scheme), che, però, viene assicurato solo su base temporanea, non risultando quindi idoneo a sopperire nel lungo periodo alla riduzione del sussidio abitativo precedentemente garantito.

Entrambe le ricorrenti affermano dunque di essere discriminate in quanto, a causa delle loro peculiari situazioni, si trovano nell'impossibilità di trasferirsi in una diversa e più piccola abitazione.

La prima ricorrente, la signora J.D., vive con la figlia gravemente disabile, di cui è caregiver primaria, e lamenta che nessuna dimora più piccola e adeguata alle necessità della figlia le è stata prospettata come valida alternativa; essa ritiene di essere discriminata sulla base della disabilità della figlia, secondo il modello della c.d. discriminazione per associazione ${ }^{11}$.

La seconda ricorrente, la signora A., invece, è una vittima di violenza di genere, perseguitata dall'ex compagno violento, da cui ha subito violenze tali da provocare in lei sintomi di disturbo post-trau-

${ }^{11}$ In un suo precedente, la Corte EDU aveva avuto modo di sancire l'applicabilità della tutela antidiscriminatoria approntata dall'art. 14 CEDU anche ai casi di discriminazione per associazione, laddove l'individuo subisca un trattamento meno favorevole non in funzione della propria disabilità, ma in forza della disabilità di un terzo a cui presta assistenza. Vedi Corte EDU, 22 marzo 2016, Guberina c. Croazia, ric. n. 23682/13. Per un'analisi di questa sentenza, sia concesso rimandare a S. FAVALLI, La Convenzione ONU sui diritti delle persone con disabilità nella giurisprudenza di Strasburgo: considerazioni a margine della sentenza Guberina c. Croazia, in "Diritti umani e diritto internazionale", n. 3, 2017, pp. 623-642. 
matico da stress, depressione e tendenze suicide, rendendola incapace di lavorare e facendola vivere in uno stato di paura costante. Quest'ultima dispone di una panic room in cui rifugiarsi in caso di aggressione, in conformità ad un progetto nazionale chiamato Sanctuary Scheme; essa lamenta di essere vittima di discriminazione sulla base del genere.

La Corte di Strasburgo esamina i ricorsi riuniti al fine di valutare se la normativa nazionale citata (Regulation B13 e DHP scheme) violi la CEDU sulla base dell' art. $14 \mathrm{CEDU}^{12}$, posto a baluardo del principio di non discriminazione, e letto in combinato disposto con l'art. 1 del Protocollo n. 1 CEDU, che tutela il diritto di proprietà. In particolare, si interroga sulla possibilità che l'intervento normativo in esame, non prevedendo un trattamento diverso a tutela delle particolari circostanze di vulnerabilità che penalizzano le due ricorrenti rispetto agli altri beneficiari del medesimo sussidio abitativo, si sostanzi in una forma di discriminazione indiretta.

${ }^{12}$ Fra i molti commenti sull'art. 14 CEDU, si vedano G.P. Dolso, F. SPITALERI, Articolo 14 , in S. Bartole, P. De Sena, V. Zagrebelsky (a cura di), "Commentario breve alla Convenzione europea per la salvaguardia dei diritti dell'uomo e delle libertà fondamentali", Padova, Cedam, 2012, p. 519 sgg.; R. O'Connell, Cinderella comes to the Ball: Article 14 and the right to non-discrimination in the ECHR, in "Legal Studies: The Journal of the Society of Legal Scholars", n. 2, 2009, pp. 211-229; J. GERARDS, The discrimination grounds of Article 14 of the European Convention on Human Rights, in "Human Rights Law Review", n. 1, 2013, pp. 99-124; O.M. ARNARDÓTTIR, The differences that make a difference: recent developments on the discrimination grounds and the margin of appreciation under Article 14 of the European Convention on Human Rights, in "Human Rights Law Review", n. 4, 2014, pp. 647-670; O.M. ARNARDÓTTIR, Cross-fertilisation, clarity and consistency at an overburdened European Court of Human Rights - the case of the discrimination grounds under Article 14 ECHR, in "Nordic Journal of Human Rights", n. 3, 2015, pp. 220-242; S. FREDMAN, Emerging from the shadows: substantive equality and Article 14 of the European Convention on Human Rights, in "Human Rights Law Review", n. 2, 2016, pp. 273-301; O.M. ARNARDÓTTIR, Vulnerability under Article 14 of the European Convention on Human Rights. Innovation or Business as Usual?, in "Oslo Law Review", n. 3, 2017, pp. 150-171; M.G. PutaTuro DonatI, Il principio di non discriminazione ai sensi dell'art. 14 CEDU: risvolti sul piano del diritto internazionale e del diritto interno, in "Europeanrights.eu", 6 maggio 2015, http://www.europeanrights.eu/index.php (consultato il 29 giugno 2020); J. GERARDS, Prohibition of Discrimination, in P. VAN DUK, F. VAN HOOF, A. VAN RUN, L. ZWAAK, "Theory and practice of the European Convention on Human Rights", Cambridge - Antwerps - Portland, Intersentia, 2018, pp. 997-1028; R. O'ConNEL, Law's Majestic Equality? Article 14, the First Protocol and Political Equality, in M. BALBONI (ed.), "The European convention on human rights and the principle of non-discrimination”, Napoli, Editoriale Scientifica, 2017, pp. 139-169. 


\subsection{Da un incipit promettente...}

La Corte EDU, innanzitutto, si chiede se il fatto che le autorità nazionali non avessero tenuto conto delle peculiari condizioni di vulnerabilità in cui le due donne - in forza rispettivamente della disabilità e del genere - versavano rispetto agli altri beneficiari del sussidio abitativo, costituisca una discriminazione nei loro confronti. Richiamando i suoi precedenti giurisprudenziali, la Corte specifica infatti che, ai sensi dell'art. $14 \mathrm{CEDU}$, può costituire discriminazione sia l'aver trattato differentemente una determinata categoria di persone, sia il non aver predisposto un trattamento differente per le stesse in forza delle loro peculiari caratteristiche ${ }^{13}$. A tal fine, occorre verificare se sussistono ragioni oggettive e ragionevoli che giustifichino il trattamento deteriore patito dalle ricorrenti, vale a dire $(i)$ se la riforma della normativa nazionale in materia di sussidi abitativi persegua un fine legittimo e (ii) se la stessa utilizzi dei mezzi proporzionati al raggiungimento di tale obiettivo.

Tuttavia, come la Corte EDU non perde occasione di ribadire, nell'operare tale valutazione, quando viene in rilievo la tutela di alcune categorie cosiddette vulnerabili - le quali necessitano quindi di una protezione rafforzata - gli Stati godono di un ridotto margine di apprezzamento nell'adottare misure che possano comprimerne i diritti ${ }^{14}$. Lo scrutinio richiesto all'autorità giudicante risulta infatti rafforzato, in quanto sono necessarie ragioni particolarmente gravi ("very weighty reasons") per giustificare una disparità di trattamento fondata su tali motivi ${ }^{15}$, fra cui rientrano anche la disabilità e il genere ${ }^{16}$.

${ }^{13}$ Corte EDU, J.D. e A. c. Regno Unito, cit., par. 85.

${ }^{14}$ Corte EDU, J.D. e A. c. Regno Unito, cit., parr. 87-89.

${ }^{15}$ Corte EDU, J.D. e A. c. Regno Unito, cit., par. 97.

${ }^{16}$ Non basta dunque il criterio adottato dalla Corte Suprema inglese, il c.d. "manifestly without reasonable foundation test", ma deve essere utilizzato il più stringente "weighty reasons test". Vedi A. TYRER, JD and A v United Kingdom, in "Stammeringlaw", 4 marzo 2020, in https://www.stammeringlaw.org.uk/jd-and-a-v-united-kingdom/ (consultato il 4 agosto 2020). Come la Corte EDU ha modo di specificare, tale criterio meno restrittivo poteva essere applicato anche nei confronti di soggetti appartenenti a gruppi vulnerabili in casi, come il precedente di Strasburgo cui si riferisce la Corte suprema inglese (Corte EDU, Stec e altri c. Regno Unito, ricorsi n. 65731/01 e 65900/01, 12 aprile 2006) in cui la differenza di trattamento era frutto di una misura solo transitoria di sicurezza sociale, volta ad appianare una forma di diseguaglianza storica. Vedi Corte EDU, J.D. e A. c. Regno Unito, 
Sulla scorta di tali promettenti premesse, la Corte EDU si appresta quindi a verificare se sussistano ragioni oggettive e ragionevoli che giustificano il trattamento deteriore subito dalle ricorrenti in forza della riforma legislativa approntata dalle autorità nazionali. La questione in esame si gioca tutta, in ultima analisi, sulla compatibilità con i valori della CEDU del sistema stesso dei sussidi abitativi nazionale, chiara espressione della criticata politica di austerità adottata negli ultimi anni dal Regno Unito.

\section{2. ...ad una conclusione deludente}

Il ragionamento della Corte di Strasburgo, che fino a questo momento aveva trattato congiuntamente i ricorsi, si bipartisce, arrivando a conclusioni diametralmente opposte per le due donne. I giudici europei rinvengono infatti una violazione della CEDU solo nel caso della seconda ricorrente, la signora A., ma non nei confronti della prima, la signora J.D.

Con riferimento a quest'ultima, la Corte EDU rileva che il trattamento deteriore da essa patito sia giustificato da ragioni oggettive e ragionevoli, quindi non discriminatorio. In primo luogo, afferma che la riforma dei sussidi abitativi persegue un fine legittimo anche alla luce delle peculiari esigenze delle persone con disabilità e dei loro caregiver. Pur riconoscendo le oggettive difficoltà della donna e della figlia disabile a trovare una diversa dimora, infatti, la Corte ritiene che l'intervento legislativo sotto esame non si pone essenzialmente in contrasto con la necessità delle persone con disabilità ad adeguate soluzioni abitative, in quanto non sussiste per gli stessi un'esigenza medica ("medical need") ad occupare un'abitazione con una stanza in più ${ }^{17}$. In seconda battuta, nota come sussistano ragioni particolarmente gravi ("very weighty reasons") a dimostrare che l'intervento normativo in parola utilizza dei mezzi proporzionati al raggiungimento del suo obiettivo di razionalizzazione

cit., par. 88. Come nota Ewing, tale specificazione potrebbe avere delle conseguenze significative per i futuri sviluppi della giurisprudenza della Corte Suprema inglese in casi di tutela antidiscriminatoria in materia di sicurezza sociale. Vedi A. EwING, "Bedroom tax" unlawful - Strasbourg Court, cit.

${ }^{17}$ Corte EDU, J.D. e A. c. Regno Unito, cit., par. 101. 
della spesa pubblica. Tali weighty reasons sono rappresentate dall'esistenza di uno strumento di sicurezza sociale alternativo, il c.d. DHP scheme, che la ricorrente può richiedere, tanto più che ne ha beneficiato in pendenza di giudizio ${ }^{18}$. La Corte si premura anche di specificare che, nonostante quest'ultimo non fornisca le stesse certezze del sistema preriforma - viene invero erogato solo su base temporanea - tuttavia è improbabile che le autorità nazionali non continuino a rinnovarlo periodicamente nei confronti della ricorrente, in forza del deterrente che la possibile applicazione dello Human Rights Act ${ }^{19}$ e del Public Sector Equality Duty ${ }^{20}$ costituiscono nell' ordinamento inglese.

In relazione alla seconda ricorrente, invece, la Corte EDU nota come il seppur legittimo obiettivo della riforma del sistema dei sussidi abitativi risulti in contrasto con quello del progetto nazionale di tutela delle vittime di violenza domestica chiamato Sanctuary Scheme. Rileva quindi come non vi siano weighty reasons a giustificazione del prevalere delle ragioni del primo intervento normativo sul secondo, alla luce del fatto che il DHP scheme non è in grado di rendere la riforma del sistema dei sussidi abitativi un mezzo proporzionato rispetto alla peculiare categoria delle donne vittime di violenza domestica, per le quali l'unico strumento di tutela consiste nella possibilità di rimanere nel luogo protetto creato grazie al progetto nazionale dedicato ${ }^{21}$.

La decisione, che va a toccare un tema estremamente delicato per i giudici di Strasburgo, quale il grado di "intromissione" che la Corte EDU può esercitare nel giudicare le politiche di sicurezza sociale adottate dagli Stati membri, non è presa all'unanimità. I giudici Peichal e Wojtyczec esprimono in particolare nella loro opinione parzialmente dissenziente alcune perplessità in merito all'argomentazione della Corte con riferimento alla doglianza della seconda ricorrente. Essi criticano il modo con cui la maggioranza ha deciso a quale di due atti legislativi nazionali con obiettivi confliggenti dare priorità, senza basare

${ }^{18}$ Corte EDU, J.D. e A. c. Regno Unito, cit., par. 102.

19 Vedi supra, nota 8.

${ }^{20}$ Il Public Sector Equality Act, introdotto dalla sezione 149 dell'Equality Act ed entrato in vigore nel 2011, impone alle autorità pubbliche nazionali di favorire la parità di opportunità ed eliminare la discriminazione fondata sui motivi enunciati, fra cui rientrano anche il genere e la disabilità.

${ }^{21}$ Corte EDU, J.D. e A. c. Regno Unito, cit., parr. 104-105. 
il proprio ragionamento sui valori sanciti dalla CEDU, addentrandosi così indebitamente nella sfera di politica legislativa propria delle autorità nazionali. In tal senso, i due giudici - in maniera condivisibile - rilevano come la situazione delle due donne dovesse essere sottoposta al medesimo ragionamento giuridico. Tuttavia, essi arrivano ad una conclusione diametralmente opposta rispetto a quella prospettata nel presente scritto, laddove fanno intendere come non fosse rilevabile in nessuna delle due situazioni una violazione della CEDU.

\section{Il "tassello mancante": il principio di non discriminazione fondata sulla disabilità}

A parere di chi scrive, sia la sentenza sia le opinioni parzialmente dissenzienti ad essa allegate sono mancanti nella parte in cui non considerano la necessità di interpretare l'art. 14 CEDU alla luce del divieto di discriminazione fondata sulla disabilità di cui alla Convenzione delle Nazioni Unite sui diritti delle persone con disabilità del 2006, così come interpretato dal correlato Comitato delle Nazioni Unite sui diritti delle persone con disabilità (o Comitato CDPD) nel suo General Comment in materia 22.

Tale trattato, infatti, che è stato ratificato dalla quasi totalità dei paesi membri del Consiglio d'Europa ${ }^{23}$, fra cui lo Stato resistente, viene ormai preso espressamente come standard di riferimento in tutte le iniziative politiche a tutela delle persone con disabilità della stessa organizzazione ${ }^{24}$. La CDPD esercita inoltre una profonda influenza sulla giurisprudenza della Corte EDU in materia di tutela delle persone con disabilità, che, in assenza di qualsiasi riferimento alla disabilità nel testo convenzionale, si è sviluppata esclusivamente in via giurisprudenziale ${ }^{25}$. A seguito dell'entrata in vigore della CDPD, i giudici di Stra-

22 Vedi, in argomento, Comitato CDPD, General Comment No. 6 (2018) on equality and non-discrimination, CRPD/C/GC/6, 26 aprile 2018.

${ }^{23}$ Ad oggi, solo il Liechtenstein non ha ratificato la CDPD.

${ }^{24} \mathrm{Da}$ ultimo, vedi la strategia del Consiglio d'Europa sulla disabilità attualmente in vigore: Council of Europe Disability Strategy 2017-2023 - Human rights: a reality for all, adottata il 30 novembre 2016.

25 V. ZAMBRANO, Il favor dei giudici di Strasburgo verso la protezione dei diritti delle 
sburgo hanno mostrato di aderire al modello di uguaglianza imposto da questa Convenzione ${ }^{26}$, arrivando addirittura ad affermare l'esistenza di un "consenso generale a livello europeo e mondiale" che sancisce appunto il divieto di discriminazione fondato sulla disabilità ${ }^{2}$.

\subsection{La discriminazione fondata sulla disabilità nella Convenzione delle Nazioni Unite sui diritti delle persone con disabilità: una breve panoramica}

Come noto, i princìpi di uguaglianza e di non discriminazione, fra loro strettamente connessi, sono alla base stessa del diritto internazionale dei diritti umani ${ }^{28}$, tanto che trovano ampia menzione sia nella Carta delle Nazioni Unite, sia in tutti i principali strumenti giuridici internazionali e regionali a tutela dei diritti fondamentali.

La Convenzione sui diritti delle persone con disabilità del 2006 si

persone con disabilità nel quadro dell'interpretazione evolutiva della CEDU, in "Studi in memoria di Maria Rita Saulle", Napoli, Editoriale Scientifica, 2014, pp. 1657-1676.

26 In proposito, sia permesso rimandare a S. FAVALLI, The United Nations Convention on the Rights of Persons with Disabilities in the case law of the European Court of Human Rights and in the Council of Europe Disability Strategy 2017-2023: "from Zero to Hero", in "Human Rights Law Review", n. 3, 2018, pp. 517-538. Vedi inoltre D. FERRI, A. BRODERICK, The European Court of Human Rights and the Human Rights Model of Disability: Convergence, Fragmentation and Future Perspectives, in "European Yearbook on Human Rights", 2019, pp. 261-294; A. BRODERICK, A reflection on substantive equality jurisprudence: The standard of scrutiny at the ECtHR for differential treatment of Roma and persons with disabilities, in "International Journal of Discrimination and the Law", 2014, pp. 1122; D. BJöRGVInsson, The Protection of the Rights of Persons with Disabilities in the Case Law of the European Court of Human Rights, in O.M. ARNAdótTIR, G. QuinN, "The UN Convention on the Rights of Persons with Disabilities, European and Scandinavian perspectives", Leiden - Boston, Brill, 2009, pp. 141-161; J. ClIFFORD, The UN disability convention and its impact on European equality law, in "The Equal Rights Review", 2011, pp. 11-25.

${ }^{27}$ Corte EDU, 30 aprile 2009, Glor c. Svizzera, ric. n. 13444/04, par. 53.

${ }^{28}$ Fra i molti, S. FARRIOR, Equality and Non-Discrimination under International Law: Volume II, Abingdon-Oxon, Routledge, 2015. Vedi inoltre COMITATO PER I DIRITTI UMANI, General Comment No. 18: Non-discrimination, 10 novembre 1989, HRI/GEN/1/Rev.1, 29 giugno 1994, p. 26; COMITATO SUI DIRITTI ECONOMICI, SOCIALI E CULTURALI, General Comment No. 20 Non-discrimination in economic, social and cultural rights (art. 2, para. 2, of the International Covenant on Economic, Social and Cultural Rights), 2 luglio 2009, $\mathrm{E} / \mathrm{C} .12 / \mathrm{GC} / 20$. 
inserisce in questo stesso filone, dando grande rilievo a tali concetti fondamentali, che permeano l'intero trattato. In essa, uguaglianza e non discriminazione sono qualificate sia come princìi, sia come diritti, rispettivamente agli artt. 3 e 5 CDPD. Inoltre, come dimostra il fatto che nel testo convenzionale venga largamente utilizzata l'espressione "su base di uguaglianza con gli altri”, tali concetti fungono da parametro interpretativo degli altri diritti sostanziali riconosciuti in capo alle persone con disabilità. Sono difatti considerati i capisaldi della tutela dei diritti delle persone disabili, costituendo degli obblighi trasversali a realizzazione immediata 29.

A questi si aggiunge il correlato obbligo di garantire accomodamenti ragionevoli ${ }^{30}$, che viene teorizzato come un autonomo diritto per la prima volta proprio nella $\mathrm{CDPD}^{31}$. Quest'ultimo concetto, infatti, costituisce nella convenzione del 2006 il "perno" per garantire l'eguaglianza sostanziale delle persone con disabilità 32 . Il rifiuto di fornire un accomodamento ragionevole - definito dall'art. 2 CDPD come l'insieme delle misure e degli adattamenti che non impongano un carico sproporzionato o eccessivo, ma sono necessari ad assicurare alle persone con disabilità il godimento e l'esercizio, su base di eguaglianza con gli altri, di tutti i diritti umani e le libertà fondamentali - viene espressamente identificato come una forma di discriminazione fondata sulla disabilità vietata dalla convenzione.

In tal senso, i princìpi di uguaglianza e non discriminazione enun-

${ }^{29}$ Comitato CDPD, General Comment No. 6 (2018), cit., par. 12.

${ }^{30}$ Fra i molti, R. BROwn, J.E. LORD, The Role of Reasonable Accommodation in Securing Substantive Equality for Persons with Disabilities: The UN Convention on the Rights of Persons with Disabilities, in M. Rioux, L.A. BASSER, M. Jones (eds.), "Critical Perspectives on Human Rights and Disability Law", 2011, Leiden - Boston, Martinus Nijhoff Publishers, pp. 273307; F. MÉGRET, D. MsIPA, Global Reasonable Accommodation: How the Convention on the Rights of Persons with Disabilities Changes the Way We Think About Equality, in "South African Journal on Human Rights", n. 2, 2014, pp. 252-271; A. Lawson, Reasonable Accommodation in the Convention on the Rights of Persons with Disabilities and Non-Discrimination in Employment: Rising to the Challenge?, in C. O'MAHONY, G. QuINN, Disability Law and Policy. An Analysis of the UN Convention, cit., pp. 359-374.

${ }^{1}$ R. Cera, Article 5, in V. Della Fina, R. Cera, G. Palmisano (eds.), "The United Nations Convention on the rights of Persons with disabilities: A Commentary, Cham, Springer, 2017, p. 167 sgg..

32 D. FERRI, L'accomodamento ragionevole per le persone con disabilità in Europa: dal Transatlantic Borrowing alla Cross-Fertilization, in "Diritto Pubblico e Comparato Europeo", n. 2, 2017, pp. 381-420. 
ciati nella CDPD rappresentano un'evoluzione dell'approccio adottato dai precedenti trattati delle Nazioni Unite sui diritti umani ${ }^{3}$. La convenzione fa riferimento ad un nuovo modello di "uguaglianza inclusiva" ("inclusive equality"), il quale va a ridefinire il concetto di uguaglianza sostanziale individuandone e definendone le differenti dimensioni, fra loro complementari: redistributiva, di riconoscimento, partecipativa, di accomodamento ${ }^{34}$. Più precisamente, la dimensione "redistributiva" indica la necessità di colmare gli svantaggi socioeconomici a cui le persone con disabilità sono sottoposte; quella "di riconoscimento" impone di affermare la loro dignità intrinseca combattendo pregiudizi e stereotipi; quella "partecipativa" sancisce l'importanza di garantire che le persone con disabilità siano membri effettivi della società; mentre quella "di accomodamento" si riferisce "all'accomodamento ragionevole come passepartout per l'esercizio dei diritti previsti dalla CDPD"35.

\section{Una (tentata) rilettura della sentenza alla luce del principio di non discriminazione fondata sulla disabilità}

Come anticipato, quel che più stupisce della sentenza in esame è che la Corte EDU non manca di richiamare la Convenzione sui diritti delle persone con disabilità del 2006 nella parte dedicata alla normativa internazionale rilevante ${ }^{36}$, né di citare alcuni suoi significativi precedenti giurisprudenziali in materia di discriminazione fondata sulla disabilità, i quali adottano il modello di uguaglianza di cui alla $\mathrm{CDPD}^{37}$. Ciononostante, di quest'ultimo non vi è traccia nell'argomentazione dei giudici di Strasburgo, che dimostrano così di aderire solo formal-

${ }^{33}$ Comitato CDPD, General Comment No. 6 (2018), cit., par. 5.

${ }^{34}$ Comitato CDPD, General Comment No. 6 (2018), cit., par. 11.

35 D. FERRI, La giurisprudenza costituzionale sui diritti delle persone con disabilità e lo Human Rights Model of Disability: "convergenze parallele" tra Corte costituzionale e Comitato ONU sui diritti delle persone con disabilità, in "Dirittifondamentali.it", n. 1, 2020, in http://dirittifondamentali.it/ (consultato il 4 marzo 2020).

${ }^{36}$ Corte EDU, J.D. e A. c. Regno Unito, cit., parr. 47-49. In particolare, la Corte EDU fa riferimento all'art. $28 \mathrm{CDPD}$ in materia di adeguati livelli di vita e protezione.

37 Il riferimento è a Corte EDU, Glor c. Svizzera, cit., e a Corte EDU, 22 marzo 2016, Guberina c. Croazia, cit. 
mente alla tutela antidiscriminatoria stabilita nella Convenzione delle Nazioni Unite, eludendo del tutto il concetto di "uguaglianza inclusiva", con particolare riferimento alle dimensioni di accomodamento e redistributiva, tanto da far dubitare dell'esistenza delle ragioni oggettive e ragionevoli che, secondo i giudici europei, dovrebbero giustificare il trattamento deteriore subito dalla ricorrente J.D.

Il modello di "uguaglianza inclusiva" della CDPD sancisce, infatti, il dovere in capo agli Stati membri di adottare quegli accomodamenti ragionevoli che siano necessari per garantire l'eguaglianza sostanziale delle persone con disabilità rispetto agli altri consociati (dimensione di accomodamento). Al fine di soddisfare tale obbligo, le autorità nazionali sono quindi tenute a valutare, in base alle circostanze del singolo caso concreto, se gli accomodamenti richiesti impongano un carico sproporzionato o eccessivo per l'amministrazione nel suo insieme, seguendo i criteri suggeriti in proposito dal Comitato $\mathrm{CDPD}^{38}$.

A parere di chi scrive, dunque, risulta criticabile l'argomentazione della Corte EDU, la quale liquida la questione notando come, nel caso concreto, nonostante per la ricorrente un forzato trasloco avrebbe effetti "extremely disruptive and highly undesirable", tuttavia non ci sono "ragioni mediche" a suffragio della necessità di un accomodamento ragionevole che preveda per la stessa una stanza ulteriore ${ }^{39}$. I giudici di Strasburgo, infatti, si soffermano su un presunto dato medico (di cui oltretutto non viene data alcuna prova, né ulteriore specificazione) che sembra riferirsi alla sola dimensione dell'accomodamento delle necessità di accessibilità degli spazi architettonici. Non tengono invece conto della situazione del singolo individuo con disabilità, per il quale lasciare la propria abitazione ben potrebbe avere un impatto negativo sulla vita di relazione, la partecipazione in società e, di conseguenza, sul benessere psicologico e mentale dell'individuo.

Un simile approccio appare per di più in pieno contrasto con i fondamenti teorici stessi della Convenzione delle Nazioni Unite, che si propone di superare l'ormai obsoleto c.d. modello medico, in favore del c.d. modello sociale. Nel primo, infatti, la disabilità è identificata meramente dalla menomazione individuale derivante da una malattia, un trauma,

${ }^{38}$ Comitato CDPD, General comment No. 6 (2018), cit., par. 26.

${ }^{39}$ Corte EDU, J.D. e A. c. Regno Unito, cit., par. 101. 
una condizione di salute che danneggia le funzioni fisiche o cognitive dell'individuo ${ }^{40}$, mentre nel secondo la disabilità è invece una costruzione sociale ${ }^{41}$, che si concretizza nella perdita o limitazione delle opportunità di prendere parte alla vita della comunità su basi paritarie con gli altri consociati a causa delle barriere ambientali e sociali presenti.

Del resto, è lo stesso rapporto periodico presentato dal Comitato CDPD in riferimento all'attuazione della Convenzione delle Nazioni Unite da parte del Regno Unito nel 201742 - di cui peraltro la Corte EDU ha contezza, visto che viene citato in sentenza - a rilevare come la legislazione antidiscriminatoria nazionale non sembri prevedere un'adeguata tutela nei confronti delle persone con disabilità, in particolare proprio in relazione al rispetto del diritto all'abitazione di queste ultime ${ }^{43}$, già messo a repentaglio dalle misure di austerità adottate dallo Stato ${ }^{44}$.

Alla luce di tali considerazioni, non risulta che la riforma del sistema nazionale dei sussidi abitativi utilizzi dei mezzi proporzionati in relazione al modello di "uguaglianza inclusiva" stabilito dalla CDPD, non rispettando l'obbligo imposto agli Stati membri di tener conto della necessità di colmare gli svantaggi socioeconomici a cui le persone con disabilità sono sottoposte in tutte le misure da essi adottate (dimensione redistributiva). Di conseguenza, non possono rinvenirsi weighty reasons a giustificazione delle misure di austerità sotto scrutinio, la cui adeguatezza è stata messa in discussione persino sul piano internazionale dallo stesso Comitato CDPD.

Inoltre, appare affrettato, se non semplicistico, affermare che il c.d. DHP Scheme, che integra la riduzione del sussidio della riforma su base temporanea, verrà sempre garantito alla ricorrente in forza dell'appli-

40 "Disability is an individual deficit deriving from a disease, trauma or health condition that impairs or disrupt physiological or cognitive functions", in C.E. DRUM, "Models and Approaches to Disability", in C.E. DruM, G.L. KRAHN, H. JR. BERSANI, Disability and Public Health, American Public Health Association/American Association on Intellectual and Developmental Disabilities, 2009, p. 27 sgg.

${ }^{41}$ M. Oliver, Understanding Disability: From Theory to Practice, Basingstoke: Macmillan, 1996, p. 35 sgg.

${ }^{42}$ Comitato CDPD, Concluding observations on the initial report of the United Kingdom of Great Britain and Northern Ireland, 3 ottobre 2017, CRPD/C/GBR/CO/1.

${ }^{43}$ Comitato CDPD, Concluding observations on the initial report of the United Kingdom of Great Britain and Northern Ireland, cit., par. 14.

${ }^{44}$ Comitato CDPD, Concluding observations on the initial report of the United Kingdom of Great Britain and Northern Ireland, cit., par. 58. 
cazione della normativa nazionale, ed in particolare dello Human Rights Act e del Public Sector Equality Duty. Se è vero infatti che, nel malaugurato caso in cui le autorità nazionali non dovessero rinnovare la misura di sicurezza sociale temporanea, la donna potrebbe adire le corti nazionali sulla base di tali strumenti normativi, è quantomeno opinabile la reale efficacia di questo sistema di tutela nel caso di specie. Basti pensare che la tutela garantita dal primo degli strumenti citati può essere attivata solo dalle Corti superiori dopo un iter processuale di certo non in grado di fornire una pronta risposta ad una persona già in situazione di grave svantaggio socio-economico.

In conclusione, l'impressione generale è che i giudici di Strasburgo abbiano incentrato il loro ragionamento sul delicato tema del grado di pervasività che la Corte EDU può esercitare nel giudicare le politiche di sicurezza sociale adottate dagli Stati membri, perdendo di vista alcuni valori fondanti della CEDU stessa. Fra questi, risulta assente appunto il principio di non discriminazione fondata sulla disabilità, letto alla luce del modello di "uguaglianza inclusiva" della Convenzione delle Nazioni Unite sui diritti delle persone con disabilità, che costituisce ormai pacificamente lo standard di riferimento per il Consiglio d'Europa in materia di tutela delle persone con disabilità. Così facendo, i giudici europei hanno perso un'ottima occasione per fornire alle autorità nazionali le indicazioni utili a fornire gli accomodamenti necessari a rendere le misure di austerità adottate in materia di sussidi abitativi compatibili con la tutela dei diritti delle persone con disabilità approntata a livello internazionale ed europeo.

\footnotetext{
Abstract - On several occasions, the austerity measures implemented by many European countries following the well-known economic crisis of 2008 have come under the scrutiny of the Strasbourg Court for their alleged violation of the fundamental rights enshrined in the European Convention of Human Rights. In particular, in the recent decision J.D. and A. v. United Kingdom the European Court, for the first time, condemned the UK's austerity policy on housing subsidies as discriminatory against the victims of do-
}

mestic violence, considering their vulnerable status. However, this ruling also represents a missed opportunity for the Strasbourg judges to guarantee the same protection to another category deserving an enhanced protection, namely persons with disabilities and, by association, their caregivers. Against this background, this paper focuses on the "missing piece" in the legal reasoning of the European Court, while attempting an analysis of the case in light of the principle of non-discrimination on the ground on disability. 